\title{
Abundances of heavy metals and lead isotopic ratios in subluminous $B$ stars
}

\section{S. O'Toole}

Dr. Remeis-Sternwarte, Astronomical Institute, University of Erlangen-Nürnberg, Sternwartstr.

7, D96049 Bamberg, Germany

and AAO Epping, Australia

E-mail: otoole@aao.gov.au

\section{U. Heber*}

Dr. Remeis-Sternwarte, Astronomical Institute, University of Erlangen-Nürnberg, Sternwartstr.

7, D96049 Bamberg, Germany

E-mail: heberdsternwarte.uni-erlangen.de

Subluminous B stars (sdB) are core helium burning stars of about half a solar mass. A new class of non-radial pulsators has recently been discovered amongst them at temperatures between $29000 \mathrm{~K}$ and $35000 \mathrm{~K}$. The richness of the pulsation modes makes these stars ideal targets for asteroseismology. We present a detailed abundance analysis of high-resolution ultraviolet echelle spectra of five subdwarf B stars obtained using the Space Telescope Imaging Spectrograph on board the Hubble Space Telescope. The goal of our observations was to test the hypothesis that pulsations in sdBs are correlated to the surface abundances of iron-group elements. We study two pulsators and three non-pulsators. We determined abundances for 25 elements including the iron group and even heavier elements such as tin and lead using LTE curve-of-growth and spectrum synthesis techniques and find strong enrichment of heavy elements up to 2.9 dex with respect to solar which are probably caused by atomic diffusion processes. We find that there is no clear correlation between pulsations and metal abundances. This poses a serious challenge to the diffusion and pulsation theory.

Abundances for the lead isotopes are derived from very high resolution spectra using an UV line of triply ionised lead. As $\mathrm{Pb}$ terminates the s-process sequence its isotopic abundance ratios yield important constraints for understanding stellar nucleosynthesis. It is very difficult to measure them in hot stars. For the first time we were able to determine them in two subluminous B stars and conclude that their ${ }^{207} \mathrm{~Pb} /{ }^{208} \mathrm{~Pb}$ are solar.

International Symposium on Nuclear Astrophysics - Nuclei in the Cosmos - IX

June 25-30 2006

CERN, Geneva, Switzerland

\footnotetext{
* Speaker.
} 


\section{Introduction}

The subdwarf B (sdB) stars are core helium-burning objects with envelopes that are too thin to sustain nuclear burning. They can be identified with models of extreme horizontal branch (EHB) stars; in other words, they have masses $\sim 0.5 M_{\odot}$ and will evolve directly to the white dwarf cooling curve, bypassing the asymptotic giant branch.

The possibility of pulsations in $\mathrm{sdB}$ stars was theoretically predicted by [1] at around the same time they were observed by [2]. The more than 30 known pulsators (officially known as V361 Hya stars) have $T_{\text {eff }}=29000-35000 \mathrm{~K}$ and $\log g=5.2-6.0$, periods of $1-10$ minutes and amplitudes less than $60 \mathrm{mmag}$. The richness of the pulsation modes makes these stars ideal targets for asteroseismology.

The driving mechanism of the oscillations is believed to be related to the ionisation of iron and other heavy elements at the base of the photosphere. As is the case for other types of pulsators there is an overlap in the $\left(T_{\text {eff }}, \log g\right)$ plane between pulsators and non-pulsators. Diffusion calculations by [3] suggest that the surface iron abundance of pulsators should be higher than that of non-pulsators, however studies by [4] and [5] find that iron has approximately solar abundance in most sdBs.

For this reason we set out to determine if any correlation exists between surface abundances of iron-group elements for pulsators and non-pulsators. Since elements such as nickel, manganese and chromium are not normally accessible through ground-based optical spectra, it was necessary to acquire high-resolution UV echelle spectra with the Space Telescope Imaging Spectrograph on board the Hubble Space Telescope (HST/STIS). Section 2 describes the spectral analysis technique used, while Sect. 3 summarises the results of the abundance analysis already published by [6]. We also report new results on the lead isotopic ratio, the first detection and analysis of a metal isotopic ratio in a hot subluminous star. Measurements of the $\mathrm{Pb}$ isotopic ratios are important to understand neutron capture nucleosynthesis both via the s-process and via the r-process.

\section{Spectral line fits}

As input for our spectrum synthesis, we used a metal line-blanketed LTE model atmosphere with solar metalicity and Kurucz' ATLAS6 Opacity Distribution Functions (for details see [5]). The spectra were synthesised using Lemke's updated version of the LINFOR program [7]. Oscillator strengths were taken from the Kurucz line list, as were damping constants for all metal lines. In the case of species heavier than $\mathrm{Zn}$, values were taken from the resonance line lists of [8,9]. Only lines that have been observed experimentally were used, since we required the most accurate wavelengths possible. For the partition functions of Ga III, Ge IV, Sn IV and Pb IV we used these ions' ground state statistical weight, since no published data are available. This is a good approximation at temperatures of $30000-35000 \mathrm{~K}$.

\section{Abundances}

The results of the quantitative spectral analysis of our HST UV echelle spectra are plotted in Fig. 1. Out of the five hot sdB stars two are member of the short-period, pulsating V361 Hya class.

Abundances of no less than 25 elements including the iron group and even heavier elements such as tin and lead have been determined. The errors range from 0.1 dex to 0.6 dex, with an 
average of 0.3 dex depending on the number and strength of available lines, degree of crowding, and quality of oscillator strengths. There is generally excellent agreement between abundances from different ionisation sages (for details see [6]).

As has been found by many previous studies, carbon abundances range from virtually none at all to slightly below the solar value while the $\mathrm{N}$ abundances are slightly below the solar value.

While iron is found to be nearly solar (PG 1219+534, Feige 48, CPD $-64^{\circ} 481$ ), slightly depleted in $\mathrm{CD}-24^{\circ} 731$ and subsolar in Feige 66 by a factor of ten, all other elements of the iron group are enhanced by between 0.5 and 2.5 dex with respect to solar values. The enhancements are large in Feige 66 and PG 1219+534, but mild for the three others. The heavy metals Ga, Ge, $\mathrm{Sn}$ and $\mathrm{Pb}$ are all enriched with respect to the Sun in all stars, reaching as high as 2.9 dex for $\mathrm{Ga}$ in PG $1219+534$ or 2.75 dex for $\mathrm{Pb}$ in Feige 66. These peculiar abundance patterns are probably caused by atomic diffusion processes in the atmosphere.

We have compared a hot pulsator (PG 1219+534) with two non-pulsators with similar stellar parameters (Feige 66 and $C D-24^{\circ} 731$ ) and a cooler pulsator (Feige 48) with a similar non-pulsator (CPD -64 481 ), and found no consistent differences between the members of each pair.

The heavy element abundance pattern of CD $-24^{\circ} 731$ comes close to that of PG $1219+534$ except for its low iron and nickel. Feige 66 has an even lower iron abundance, but its heavy metal abundance pattern does not match that of PG 1219+534 at all. In other words the abundance patterns of two non-variable stars of similar temperature and gravity are too dissimilar for a conclusive comparison with a pulsator. This finding poses a serious challenge for the theory of both pulsation and diffusion and leads us to suspect that there must be another, as yet unknown, discriminating factor between pulsating and non-pulsating sdB stars.

\section{Lead Isotopes and the ${ }^{207} \mathrm{~Pb} /{ }^{208} \mathrm{~Pb}$ ratio}

Measuring isotope ratios in stars is of key importance in understanding the processes governing nucleosynthesis of the elements. Unfortunately such measurements are difficult in stars since there are only a handful of elements where the isotope splitting of spectral lines is large enough to be measured. The isotopes of $\mathrm{Pb}$ have been previously investigated in metal-poor halo stars as well as in a main-sequence B star in the SMC.

The lead isotope ${ }^{208} \mathrm{~Pb}$ is the terminal point of the decay of the radioactive actinide sequence - those elements that are formed only by the $r$-process. Nucleosynthesis via the $s$-process also has a final peak at ${ }^{208} \mathrm{~Pb}$. This makes lead one of the most important elements in nucleosynthesis modelling.

In metal-poor halo stars lead isotopic ratios have been measured from very few lines of neutral lead $(\mathrm{Pb} \mathrm{I})$. In hot stars, however such as $\mathrm{sdB}$ stars lead is highly ionised and the resonance lines of triply ionised lead $\mathrm{Pb}$ IV are expected to be the strongest.

Very high resolution UV spectra ( $R=114000, \mathrm{E} 140 \mathrm{H}$ grating of HST/STIS $)$ of the sdB stars Feige 66 and $\mathrm{CPD}-64^{\circ} 481$ covering the wavelength range $1160-1361 \AA$ were retrieved from the MAST archive. The spectral line of interested in the Pb IV resonance line at $1313.07 \AA$, which is one of two Pb IV resonance lines; the other is at $1028.61 \AA$, outside the spectral window of HST.

The relative contribution of the ${ }^{204} \mathrm{~Pb},{ }^{206} \mathrm{~Pb},{ }^{207} \mathrm{~Pb}$, and ${ }^{208} \mathrm{~Pb}$ to the line blend are shown in Fig. 2 (left hand panel) assuming a solar system $\mathrm{Pb}$ isotope ratio. In the right hand panel of 


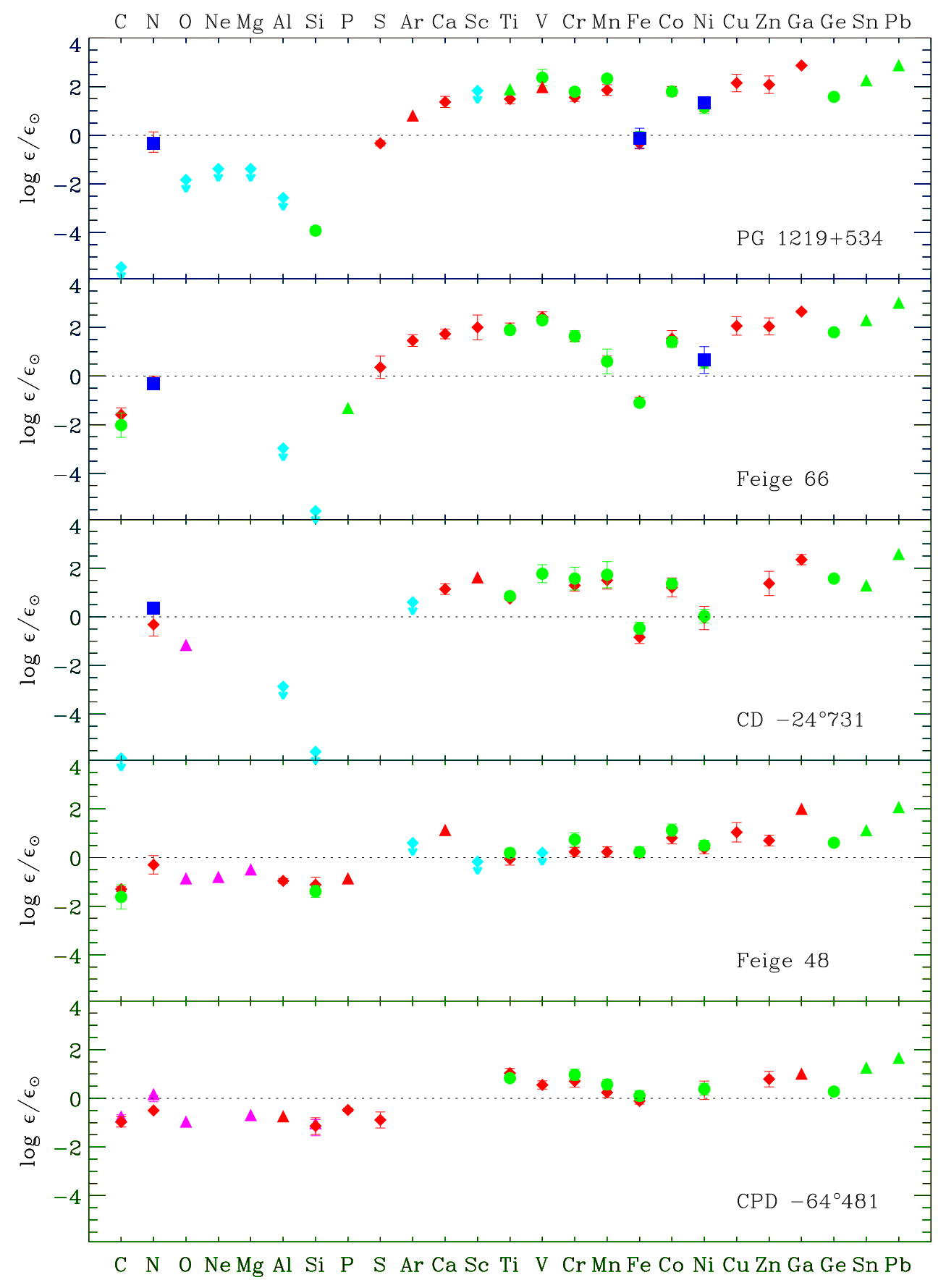

Figure 1: Abundances measured for our five targets. Magenta symbols represent values determined using singly ionised species, green represents doubly ionised species, red represents triply ionised species, blue denotes quadruply ionised species and cyan represents upper limits. Note the generally excellent agreement between different ionisation stages (from [6]). 

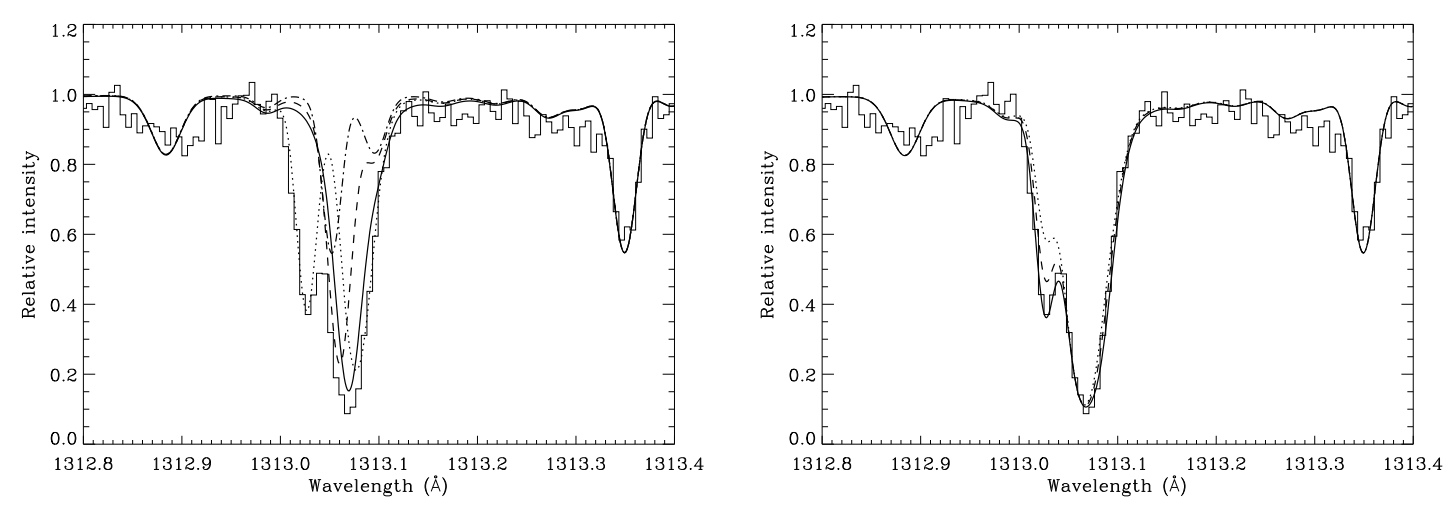

Figure 2: left hand side: The individual isotope contributions to the $\mathrm{Pb}$ IV resonance line in $\mathrm{CPD}-64^{\circ} 481$ are shown. The observed spectrum is shown as a histogram, with the isotopes shown as follows: ${ }^{204} \mathrm{~Pb}-$ dash-dotted; ${ }^{206} \mathrm{~Pb}$ - dashed; ${ }^{207} \mathrm{~Pb}$ - dotted; ${ }^{208} \mathrm{~Pb}$ - solid. As can be seen, the blue most component of the ${ }^{207} \mathrm{~Pb}$ line is easily resolved. Right hand side: The $\mathrm{Pb}$ IV line profile of CPD $-64^{\circ} 481$ is shown. Over-plotted is our model calculated with the solar system $\mathrm{Pb}$ isotope ratio, as well as 0.5 and 0.25 times the amount of ${ }^{207} \mathrm{~Pb}$.

Fig. 2 we depict the effect of reducing the ${ }^{207} \mathrm{~Pb} /{ }^{208} \mathrm{~Pb}$ ratio to subsolar values. As can be seen the ${ }^{207} \mathrm{~Pb} /{ }^{208} \mathrm{~Pb}$ ratio is solar. We cannot make any definitive statements with regard to the other isotopes. In fact, our observations are also consistent with $n o{ }^{204} \mathrm{~Pb}$. We can conclude though, that the solar system isotope mix is consistent with our observations.

\section{References}

[1] S. Charpinet, G. Fontaine, P. Brassard, B. Dorman The Potential of Asteroseismology for Hot, Subdwarf B Stars: A New Class of Pulsating Stars?, ApJ 1996 (471) L103

[2] D. Kilkenny, C. Koen, D. O’Donoghue, R. S. Stobie A new class of rapidly pulsating star - I. EC 14026-2647, the class prototype, MNRAS, 1997 (285), 640

[3] S. Charpinet, G. Fontaine, P. Brassard et al. A Driving Mechanism for the Newly Discovered Class of Pulsating Subdwarf B Stars, ApJ (483), L123

[4] H. Edelmann, U. Heber, R. Napiwotzki, Metal Abundances of sdB Stars, Baltic Astronomy 2006 (15) 103

[5] U. Heber, I. N. Reid, \& K. Werner Spectral analysis of multi mode pulsating sdB stars. II. Feige 48, KPD 2109+4401 and PG 1219+534, A\&A 2000 (363) 198

[6] S.J. O'Toole, \& U. Heber Abundance studies of sdB stars using UV echelle HST/STIS spectroscopy, $A \& A, \mathbf{2 0 0 6}$ (452) 579

[7] M. Lemke Abundance anomalies in Main Sequence A stars. I - Iron and titanium, A\&A 1989 (225) 125

[8] D.C. Morton Atomic Data for Resonance Absorption Lines. II. Wavelengths Longward of the Lyman Limit for Heavy Elements ApJS 2000 (130) 403

[9] D.C. Morton Atomic Data for Resonance Absorption Lines. III. Wavelengths Longward of the Lyman Limit for the Elements Hydrogen to Gallium, ApJS 2003 (149) 205 